\title{
EDITORIAL
}

\section{(Re)descubriendo el control de convencionalidad: ¿activismo o autocontención judicial?}

\author{
José María Goerlich Peset \\ Catedrático de Derecho del Trabajo y Seguridad Social \\ Universitat de València \\ ORCID ID: 0000-0002-2910-2153
}

doi: https://doi.org/10.20318/labos.2021.6044

\section{Las normas internacionales del trabajo en el derecho interno}

El pasado 27 de noviembre tuve la ocasión de participar, junto con Antonio Baylos y Patricia Nieto, en el tribunal que había de valorar la tesis doctoral de Miguel Ángel Garrido, dirigida por mi querida amiga Adoración Guamán. El trabajo, titulado La recepción del derecho internacional del trabajo en la jurisprudencia de los tribunales españoles, obtuvo la máxima calificación. A la espera de que los resultados de esta investigación vean pronto la luz -espero que un avance en el próximo número de esta Revista-, haber participado en su enjuiciamiento me dio la oportunidad de reflexionar sobre esta cuestión; y me empuja a compartir ahora algunas inquietudes que me suscita. Aun sin ser un experto en los diferentes aspectos implicados, observo como está adquiriendo creciente importancia tanto en nuestra reflexión teórica como, sobre todo, en la práctica cotidiana de nuestros tribunales; y no estoy seguro de que la derrota que siguen las soluciones sea siempre la más adecuada en relación con algunos valores esenciales de nuestro ordenamiento jurídico y aun de nuestra vida ciudadana.

$\mathrm{Mi}$ aportación en este terreno comienza por poner sobre la mesa dos afirmaciones que, al menos desde que yo recuerdo, nunca han sido objeto de discusión en el ámbito jurídico laboral: la importancia del llamado Derecho internacional del trabajo y la especial eficacia de las normas contenidas en los tratados internacionales. Desde que me dedico a esto, ambas ideas pueden encontrarse en cualquier manual de Derecho del Trabajo. En los que estudiábamos en los años 80 se señalaba en esta línea como "la reglamentación internacional del trabajo ha sido considerada como una condición indispensable para la existencia misma, el mantenimiento y el desarrollo de las legislaciones nacionales". En cuanto al espacio de las normas internacionales en el ámbito interno, "la Constitución admite implícitamente la superioridad de los tratados internacionales sobre las normas internas en caso de conflicto entre ellas... (art. 96.1 CE)”, lo que, por lo demás, implicaría la confirmación de lo que se había sostenido en la jurisprudencia anterior ${ }^{1}$.

En las casi cuatro décadas que han transcurrido desde entonces, las cosas han cambiado poco. Por lo que se refiere a la prevalencia de las normas internacionales, el argumento implícito que resulta del art. 96.1 CE ha sido, como es sabido, claramente confirmado por sucesivas intervenciones

\footnotetext{
${ }^{1}$ Departamento de Derecho del Trabajo Universidad de Valencia, Lecciones de Derecho del Trabajo, Valencia, 1983, pp. 56 y 120. En sentido similar, ALONSO OLEA, Derecho del trabajo, 9a ed. rev., Madrid (Publicaciones Universidad Complutense), 1985, pp. 486 y 492.
} 
normativas y jurisprudenciales. Sin ánimo de entrar en detalles al respecto, cabe traer a colación, de un lado, las previsiones de la Ley 25/2014, de 27 de noviembre, de Tratados y otros Acuerdos Internacionales. Sobre la base de las previsiones de aquel precepto (cfr. art. 28), la norma establece de forma expresa en su art. 31 que "las normas jurídicas contenidas en los tratados internacionales válidamente celebrados y publicados oficialmente prevalecerán sobre cualquier otra norma del ordenamiento interno en caso de conflicto con ellas, salvo las normas de rango constitucional". De otro, la jurisprudencia constitucional ha ratificado igualmente este planteamiento, si bien ha descartado que la "prevalencia de los tratados", a la que se refiere la rúbrica de este último precepto, constituya jerarquía normativa en sentido estricto puesto que el art. 96.1 CE "no atribuye superioridad jerárquica a los tratados sobre las leyes internas, aunque establece, de un lado, una regla de desplazamiento por parte del tratado de la norma interna anterior, sin que ello suponga su derogación, y, de otro, define la resistencia del tratado a ser derogado por las disposiciones internas posteriores en el tiempo, sin que esto último suponga la exclusión de la norma interna del ordenamiento nacional, sino su mera inaplicación" (STC 140/2018, 20 diciembre).

Seguramente la discusión de si estamos ante una relación jerárquica o de otro tipo es sumamente interesante desde una perspectiva teórica; y, desde luego, inclinarse por una u otra solución tiene consecuencias prácticas importantes como veremos de inmediato. Pero, a los efectos que aquí interesan, no creo que haga falta entrar en un análisis de este tipo. Lo que vale la pena resaltar es que la prevalencia de las normas internacionales no ha estado en discusión en las más de cuatro décadas transcurridas desde 1978. Como tampoco lo ha estado el hecho de que su garantía queda en manos de la jurisdicción ordinaria. Es verdad que, en los momentos iniciales de vigencia de la Constitución, existió la duda de si la misma correspondía en exclusiva a la constitucional, ante la que los tribunales ordinarios habrían de plantear la oportuna cuestión con base en el art. 96.1 CE, en los casos en los que una ley contradijera una disposición self-executing de un tratado en vigor ${ }^{2}$. Sin embargo, el Tribunal Constitucional descartó de forma relativamente temprana que esto fuera así3.

Con cita de pronunciamientos precedentes que se remontan a la segunda mitad de los años 80, la ya citada STC 140/2018 advierte que los tratados internacionales "no constituyen canon para el enjuiciamiento de la adecuación a la Constitución de normas dotadas de rango legal" de modo que el conflicto entre estas y aquellos no hace aparecer un conflicto de "constitucionalidad mediata" por el desconocimiento del art. 96.1 CE. De este modo, el que, por acción de la jurisprudencia de la Corte Interamericana de Derechos Humanos, conocemos como control de convencionalidad queda en manos de los tribunales ordinarios: puesto que, en línea con lo que se ha indicado más arriba, "la constatación de un eventual desajuste entre un convenio internacional y una norma interna con rango de ley no supone un juicio sobre la validez de la norma interna, sino sobre su mera aplicabilidad" se abre "una cuestión de determinación de la norma aplicable en la solución de cada caso concreto" que compete a la jurisdicción ordinaria. Así las cosas, queda "extramuros de las competencias del Tribunal Constitucional”. Otra cosa es que, a través del recurso de amparo, cupiera la revisión

${ }^{2}$ GALIANA MORENO, J., "La vigencia en España de los tratados internacionales de carácter laboral”, Revista de Política Social, 121(1979), p. 195

${ }^{3}$ Una síntesis de la evolución de la jurisprudencia constitucional sobre este aspecto en ANDRES SAENZ DE SANTAMARÍA, P., "Artículo 96", en RODRÍGUEZ-PIÑERO, M. y CASAS, M.E., Comentarios a la Constitución española. Conmemoración del XL aniversario de la Constitución, Madrid (BOE/Fundación Wolters Kluwer/Tribunal Constitucional), 2018, pp. 364 ss. Véase también CANOSA USERA, R., El control de convencionalidad, Cizur Menor (Civitas/ Thomson Reuters), 2015, pp. 84 ss.

Acaso los datos no sean tan concluyentes como se deduciría de la presentación que hace de ellos la STC 140/2018, a la que se hace refefencia inmediatamente en el texto. En este sentido, JIMENA QUESADA, L., Jurisdicción nacional y control de convencionalidad. A propósito del diálogo judicial global y de la tutela multinivel de derechos, Cizur Menor (Aranzadi/Thomson Reuters), 2013, expone con detalle las dudas que pueden deducirse de la jurisprudencia constitucional. Hay que resaltar, en todo caso, que se recoge también una extensa tradición interpretativa de la jurisdicción ordinaria en relación con la prevalencia de las normas internacionales sobre las internas. 
"de la selección del derecho formulada por los jueces ordinarios en determinadas circunstancias bajo el parámetro del artículo 24.1 CE". Pero esto solo ocurrirá "si la aplicación de la legalidad es fruto de un error patente con relevancia constitucional" o "si fuere arbitraria, manifiestamente irrazonada o irrazonable" pues entonces "no podría considerarse fundada en Derecho".

\section{La creciente importancia del control difuso de convencionalidad}

Dado que la jurisprudencia ordinaria no ha puesto en cuestión la prevalencia de los tratados internacionales desde los primeros tiempos de la vigencia de la Constitución ni su capacidad para proceder a su aplicación directa en caso de conflicto con las normas internas ${ }^{4}$, resulta curiosa la importancia que ha adquirido en los últimos tiempos en la reflexión doctrinal y en el debate judicial en el ámbito social; y, sobre todo, el cambio de signo que parece haberse introducido. Tradicionalmente, las normas internacionales de carácter laboral han tenido más bien la condición de "herramienta interpretativa de refuerzo, aclaración o integración de las correspondientes reglas internas" ${ }^{\text {. }}$. Han tenido, esto es, un papel a la hora de fijar la interpretación de las normas internas, en relación con los derechos fundamentales y libertades (art. 10.2 CE) o con otras cuestiones; y también para salvar las lagunas que presenta el ordenamiento interno. Aunque no es el único caso, cabe traer a colación como ejemplo, en este último terreno, los muchos pronunciamientos que aplican el convenio 132 OIT para completar las lagunas de la regulación en materia de vacaciones del art. $38 \mathrm{ET}^{6}$. Sin embargo, en los últimos tiempos, surge con fuerza una dimensión adicional: los órganos judiciales protagonizan un control directo de las normas legales, corrigiendo su alcance o condenándolas directamente a la inaplicación.

La STC 140/2018, y las que han reiterado su doctrina ${ }^{7}$, puede haber jugado un papel relevante en este terreno ${ }^{8}$. Pero no creo que quepa atribuirle un papel decisivo puesto que, como hemos visto, la doctrina que establece no es enteramente novedosa. Al máximo, puedo pensar que la refundición de la doctrina jurisprudencial anterior y la claridad con que se formula la posibilidad de un control difuso de convencionalidad sobre las normas legales ha tenido un impacto simbólico. Pero en mi opinión la nueva dimensión que la aplicación de las normas internacionales presenta en los últimos años tiene raíces más profundas y diría además que busca precisos objetivos en términos de política del derecho.

En las últimas décadas hemos asistido a la teorización de un sistema multinivel de protección de los derechos humanos y, entre ellos, de los derechos sociales ${ }^{9}$. Los textos internacionales que los

\footnotetext{
${ }^{4}$ Véase, por ejemplo, STS cont. 30 septiembre 1982, RJ 1982\4917, con cita de otras anteriores.

${ }^{5}$ MARTÍNEZ MORENO, C., "El marco internacional para la tutela de los derechos laborales", Lex Social, vol. 6, núm, 1/2016, p. 96, con otras referencias.

${ }^{6}$ Últimamente, STS 394/2020, 22 mayo, con cita de otras muchas, en relación con el extensamente discutido tema de la determinación de la retribución.

${ }^{7}$ La sentencia 140/2018 resuelve el recurso de inconstitucionalidad contra la reforma de la LOPJ en relación con la llamada jurisdicción universal. Con posterioridad, varias sentencias resuelven las cuestiones que habían planteado diferentes órganos jurisdiccionales al respecto y algún recurso de amparo (cfr. 10/2019, 28 enero, 23/2019, 25 febrero, 35 y 36/2019, 25 marzo, y 89/2019, 17 junio). Por lo que aquí interesa la doctrina se ha reiterado en la STC118/2019, 16 octubre, a la que me referiré en el texto un poco más adelante.

${ }^{8}$ JIMENA QUESADA, L., "La consagración del control de convencionalidad por la jurisdicción constitucional en España y su impacto en materia de derechos socio-laborales (comentario a la STC 140/2018, de 20 de diciembre)", Revista General de Derecho del Trabajo y de la Seguridad Social 53(2019).

${ }^{9}$ Un análisis panorámico de los diferentes textos en DÍAZ BARRADO, C.M., "La contribución de las normas internacionales y del derecho comunitario europeo a la configuración de un «orden social» en España”, en SEMPERE, A.V. (dir.), El modelo social en la Constitución Española de 1978, Madrid (MTAS), 2033, pp. 91 ss., o MARTÍNEZ MORENO, C., "El marco internacional para la tutela de los derechos laborales", Lex Social, vol. 6, núm, 1/2016, pp. 98 ss. Respecto a la articulación entre los diferentes niveles, MONEREO PÉREZ, J.L., "Los principios del sistema jurídico internacional multinivel de garantía de los derechos fundamentales", en Revista General de Derecho del Trabajo y de la Seguridad Social, 45 (2017)
} 
protegen a nivel global, en el marco de la ONU, o «regional» europeo, en el ámbito del Consejo de Europa o, más tarde, en el de la Unión Europea, interactúan entre ellos y con la protección que les dispensan las Constituciones nacionales. En nuestro sistema jurídico, la propia Constitución fuerza tal interacción multinivel como se advierte en el art. 10.2 CE. A ello hay que añadir que los derechos sociales en el ámbito laboral y de la protección social tienen específico reconocimiento y desarrollo a través de la actividad normativa de la OIT. Por otra parte, en los diferentes niveles existen instrumentos específicos para la «administración» de los textos correspondientes, que, según los casos, están al alcance no solo de los estados y otros sujetos del Derecho internacional sino también de las organizaciones o particulares que actúan en el ámbito interno. Comisiones, comités y, a veces, verdaderos tribunales actúan como garantes de la integridad de las normas internacionales.

A la postre, los diferentes tratados junto con la documentación que nace de estos organismos de garantía suministran un formidable arsenal a disposición de los operadores jurídicos que, en materia laboral, es particularmente extenso por la existencia de los convenios de la OIT, sus recomendaciones y sus propios órganos aplicativos. Asimismo, el sistema multinivel pone a disposición de los órganos judiciales instrumentos procesales en los que verificar los propios criterios: las cuestiones de carácter prejudicial, en vía interna ante el TC, supranacional ante el TJUE o internacional ante el TEDH, si se llegara a incorporar el protocolo 16 del Convenio Europeo de Derechos Humanos. La complejidad creciente del sistema resultante de todo ello, en el que estos instrumentos permiten aplicar normas que contienen amplias formulaciones y principios generales, posibilita giros interpretativos sobre los aspectos conflictivos. Todos tenemos en la cabeza, en este sentido, supuestos en los que la cuestión de constitucionalidad ha permitido corregir la interpretación consolidada en el TS, que en principio es supremo intérprete de la legalidad ordinaria (art. 123.1 CE). Pero, más recientemente, hemos visto la frecuencia con que la cuestión prejudicial ante el TJUE se utiliza para revisar, o intentarlo, al menos, las avanzadas en los órganos superiores de la jurisdicción ordinaria o, incluso, por el propio Tribunal Constitucional.

Por supuesto, el control de convencionalidad difuso que resulta de la interpretación consolidada del art. 96.1 CE puede jugar un papel todavía más relevante en este terreno, puesto que implica al control directo de la actuación legislativa por los jueces ordinarios, sobre la base del extenso arsenal documental que he comentado. Es verdad que, como hemos visto, nada ha cambiado recientemente desde la perspectiva formal. El sistema multinivel de protección de los derechos fundamentales así como la extensión del llamado Derecho internacional del trabajo no son aspectos que hayan aparecido de la noche a la mańana. Y, sin embargo, el despliegue del control de convencionalidad parece un fenómeno que se produce en la última década. Por eso, creo que este último fenómeno se explica desde una concreta perspectiva de política del Derecho. El control difuso de convencionalidad aparece en este sentido como una herramienta para combatir o revertir las reformas laborales y los recortes que se han producido como consecuencia de la crisis económica.

Ello explica el sustancial cambio que se ha producido en la reflexión doctrinal en relación con las reales funciones de las normas internacionales. La aproximación tradicional hacía hincapié en su escasa importancia real. Se ha llegado a afirmar que se constituyen un "tema marginal" en el tratamiento jurídico-laboral ${ }^{10}$. Aparte de que excede los confines del área de atención de los laboralistas, ha sido un lugar común en el análisis de que, aun "compartiendo en parte una misma inicial «razón de ser», cabría decir que el desarrollo del Derecho social internacional ha ido muy por detrás de los más elaborados y acabados Derechos nacionales del trabajo" ${ }^{11}$. Sin embargo, se ha pasado de entender que "los estándares mínimos que suelen contener las normas internacionales ya eran superados

\footnotetext{
${ }^{10}$ GALIANA, "La vigencia en España” cit., p. 176.

${ }^{11}$ GARCÍA-PERROTE ESCARTÍN, I., "Concepto, contenido y eficacia del derecho social internacional y del derecho social comunitario”, Revista del Ministerio de Trabajo y Asuntos Sociales, 2(1997), p. 16.
} 
-en ocasiones con creces- por la normativa laboral nacional" a considerarlos un punto central de interés en relación "con la crisis y las subsiguientes políticas de ajuste y recorte de derechos laborales y sociales" y a ser objeto de "creciente cita normativa y doctrinal en las más recientes resoluciones judiciales del orden social"12. El recurso al control de convencionalidad se relacionaría, pues, con el "cada vez... más evidente... contraste entre nuestras normas internas, progresivamente degradadas, y las normas internacionales"13.

No es de extrañar, en este contexto, que la nueva dimensión de la aplicación de las normas internacionales por nuestros tribunales, que trasciende su tradicional utilización como instrumento de integración o interpretación de la legislación interna, se proyecte fundamentalmente sobre determinados aspectos de las reformas legislativas de principios de la pasada década. Es verdad que, en el análisis que he hecho al respecto -que no es, ni mucho menos, exhaustivo-, la gama de asuntos que he detectado no se limita a la valoración multinivel de las reformas de 2010/2012. Otras cuestiones, como los criterios de valoración del ejercicio de los derechos de conciliación ${ }^{14}$ o de las pretensiones administrativas de reintegro de prestaciones indebidas ${ }^{15}$ se han sometido a confrontación con la convencionalidad. Creo, sin embargo, que cabe destacar como una parte importante de la actividad se ha centrado en cuestiones relacionadas con un elemento central en aquellas: el régimen de la extinción del contrato. A continuación, recuerdo los principales episodios en este plano.

\section{Algunos episodios en concreto}

\section{Control de la causalidad de los despidos en interés de la empresa}

El primero de los puntos que me interesa resaltar es la reconstrucción jurisprudencial del control causal del despido económico tras su reforma de 2012. La normativa anterior, desde 1994, diseñaba un modelo basado en tres elementos: en primer término, el control de hecho relacionado con la realidad de la causa aducida; en segundo lugar, el ligado a la idea de "razonabilidad" utilizada por las normas aplicables y que se vinculaba a la justificación de la medida extintiva a la luz de la causa aducida; en tercer lugar, y en fin, uno de carácter finalista que obligaba a verificar si la situación resultante del despido situaba a la empresa en una mejor situación en el mercado y permitía pronosticar la salvaguarda del empleo restante. Los problemas interpretativos que venía suscitando

\footnotetext{
${ }^{12}$ MARTÍNEZ “El marco internacional” cit., pp. 96 y 97 y 114.

${ }^{13}$ FORCADELL, A., y NAVARRO, C., "Compatibilidad del control de convencionalidad con la superación del control de constitucionalidad. Comentario a la STSJ Cataluña de 17 de enero de 2020, que declara la inaplicabilidad del art. 52 d) ET durante el período de vigencia anterior a su derogación", IusLabor 1(2020), p. 153. Esta dimensión puede verse también en LLOBERA VILA, M., "El control de convencionalidad: la aplicación de los Tratados internacionales en la determinación de estándares laborales", nueva Revista Española de Derecho del Trabajo 222(2019).

${ }^{14}$ Un nutrido grupo de pronunciamientos dictados por el TSJ de Canarias (Las Palmas) ha incorporado el criterio de convencionalidad para valorar las pretensiones en materia de conciliación (cfr. sentencia 12 marzo 2019, rec. 19/2019, cuya doctrina es reiterada en las de 27 agosto 2019, rec. 533/2019, 11 septiembre 2019, rec. 1311/2019, 17 diciembre 2019, rec. 860/2019, 15 enero 2020, rec. 1396/2019, 14 febrero 2020, rec. 1429/2019, 2 junio 2020, rec. 184/2020, o 1 septiembre 2020, rec. 197/2020).

${ }^{15}$ Me refiero a la cuestión de la posible incidencia de la doctrina del TEDH en relación con los procedimientos de revisión de prestaciones indebidamente reconocidas (art. 55). La STEDH 26 abril 2018, Cakarevic contra Croacia, consideró contraria al derecho de propiedad la ejecución de la obligación de restituir las cuantías indebidamente percibidas sin tomar en consideración la situación de necesidad en que pudiera quedar el beneficiario. En suplicación, STSJ País Vasco 2 julio 2019, rec. 1119/2019 se ha inclinado decididamente por la aplicación de la doctrina derivada del $\mathrm{CEDH}$ en la valoración de las pretensiones de reintegro ejercitadas por las Entidades Gestoras. La STSJ Cataluña 9 abril 2019, rec. 5480, dictada por el pleno de la Sala, ha excluido que la doctrina del TEDH se proyecte sobre la fase declarativa, sin perjuicio que las circunstancias tomadas en consideración por el tribunal europeo puedan ser relevantes en el momento de la ejecución de la obligación de reintegro. Aunque la doctrina posterior de la Sala ha adoptado este criterio (sentencias 27 noviembre 2019, rec. 3650/2019, 11 febrero 2020, rec. 5952/2019, o 17 julio 2020, rec. 1570/2020), la sentencia de referencia se aprobó por mayoría, existiendo un extenso y nutrido voto particular que sostiene una posición contraria.
} 
intentaron superarse en la reforma de 2010, aunque fue la de 2012 la que aspiró a cerrarlos de forma definitiva. La Exposición de Motivos del RDL 3/2012 y de la posterior Ley 3/2012, lo planteaba con total claridad al constreñir el control judicial "a una valoración sobre la concurrencia de unos hechos: las causas”. De este modo, la regulación de las causas del despido económico, a partir de 2012, incrementaría notablemente la discrecionalidad empresarial en la determinación de las medidas procedentes y, por tanto, reduciría sustancialmente el alcance de las posibilidades de verificación de jueces y tribunales.

Este planteamiento fue inicialmente aceptado por la jurisprudencia social que, sin embargo, rectificó de inmediato ${ }^{16}$. Fue la STS de 17 de julio de 2014 (rec. 32/2014) -que continúa siendo citada por el TS y los TTSSJJ ${ }^{17}$ - la que desarrolla extensamente la fundamentación del criterio del Alto Tribunal sobre el alcance del control judicial de las causas. Aunque reconoce que de la ley ha de deducirse "de manera taxativa" la limitación del control a los aspectos de hecho, sin enjuiciar en modo alguno la razonabilidad de la decisión empresarial, la "rotundidad" y la "contundencia" de las normas reformadoras en este sentido ha de entenderse como meramente "formal" -calificativo este que se utiliza dos veces en el inicio del razonamiento- pues "por fuerza ha de persistir un ámbito de control judicial fuera de la «causa» como hecho, no sólo por la concurrencia de los intereses constitucionales y compromisos internacionales que están en juego, sino también por aplicación de los principios generales en el ejercicio de los derechos". Por lo que se refiere a los compromisos internacionales, que son los que aquí interesan, el TS se refiere a los arts. 4 y 9 del Convenio 158 OIT, que respectivamente configuran el carácter causal del despido y la posibilidad de que el mismo sea objeto de revisión.

\section{Admisibilidad del contrato de apoyo a los emprendedores}

Acaso podría pensarse que en este primer episodio el TS se movió en el terreno más tradicional al que me he referido más arriba: el uso de las normas internacionales como "herramienta interpretativa" de las internas. Pero existen otros en los que las decisiones judiciales se dirigen formalmente a la remoción de las soluciones establecidas. Un caso muy claro se encuentra en la pretendida ilegitimidad del ya desaparecido contrato de apoyo a los emprendedores (art. 4 Ley 3/2012). Desde el primer momento, se discutió su admisibilidad a la luz de las normas internacionales aplicables, fundamentalmente, aunque no solo, del art. 4.4 de la Carta Social Europea ${ }^{18}$; y, una vez agotadas las vías prejudiciales, la cuestión se transformó en un tema de aplicación directa del control de convencionalidad. En el primer terreno, el Tribunal Constitucional descartó que el contrato vulnerara las reglas constitucionales (SSTC 119/2014, 16 julio, y 8/2015, 22 enero); y, poco después, el TJUE, que existiera contradicción con el art. 30 CDFUE y la Directiva 1999/70/CE del Consejo, de 28 de junio de 1999, relativa al Acuerdo marco de la CES, la UNICE y el CEEP sobre el trabajo de duración determinada (sentencia de 5 de febrero de 2015, C-117/14).

\footnotetext{
${ }^{16}$ En el primer sentido, STS 20 septiembre 2013 (rec. 11/2013). Pero ya a primeros de septiembre, STS 27 enero 2014, rec. 100/2013, inauguraba una línea interpretativa de control más intenso en relación con la modificación sustancial de condiciones de trabajo. Esta línea fue después proyectada sobre el despido de carácter económico (STS 26 marzo 2014, rec. 158/2013, y 25 junio 2014, rec. 165/2013).

${ }^{17}$ Por ejemplo, STS 232/2019, 20 marzo o SSTSJ Com. Valenciana 26 noviembre 2020, rec. 1664/2020, y Madrid 30 septiembre 2020, rec. 469/2020, 25 noviembre 2020, rec. 479/2020.

${ }^{18}$ Un análisis detallado en SALCEDO BELTRÁN, M.C., "El contrato de apoyo a emprendedores: su difícil encaje en la normativa internacional, europea y nacional”, Revista de Derecho Social 62(2013), pp. 93 ss. Las cuestiones vinculadas a la posible aplicación del convenio 158 OIT quedaron desarticuladas a raíz de la resolución de la queja presentada ante el comité: cfr. GUTIÉRREZ PÉREZ, M.: "Examen de la reforma laboral de 2012 a la luz del Convenio 158 OIT: el reciente informe de la OIT", Revista General del Derecho del Trabajo y de la Seguridad Social, 39(2014), pp. 301 ss.
} 
En el terreno del control de convencionalidad en sentido estricto, aparte los precedentes ante los JJSS ${ }^{19}$, el primer pronunciamiento de suplicación es la STSJ Cataluña 22 junio 2015, rec. $134 / 2015$, en la que el pleno de la Sala aborda frontalmente este problema. La mayoría de la sala excluyó que el art. 4.4 CSE fuera una norma self executing, y que resultaran vinculantes los pronunciamientos del Comité de derechos sociales en relación con el tema. Adicionalmente, el pronunciamiento indicaba que, de existir vulneración entre el art. 4 Ley 3/2012 y la Carta Social Europea, no implicaría la nulidad del período de prueba en la medida en que se sobrepasaran los límites del art. 14 ET sino únicamente la necesidad de indemnizar por falta de preaviso.

Es importante señalar que el pronunciamiento va acompañado por un extenso voto particular, suscrito por un nutrido grupo de magistrados, que se mueve en una línea diferente: sobre la base del art. 4.4 CSE y la doctrina del CEDH habría de concluirse en la existencia de contradicción entre la norma interna y la internacional y dar prevalencia a esta última con la consecuencia de considerarla aplicable. Y es importante hacerlo porque el mismo ha tenido cierta importancia en la aplicación judicial del art. 4 Ley 3/2012. En efecto, si bien es cierto que buena parte de los TTSSJJ se han inclinado por una solución defendida por la mayoría ${ }^{20}$, no lo es menos que una porción no desdeńable han aceptado los postulados interpretativos sostenidos en el indicado voto particular, incluso convirtiéndolo explícitamente en fundamento de las decisiones, aunque con la salvedad de que los efectos que se aparejan a la vulneración de la Carta Social Europea se ciñen a la indemnización por preaviso y no alcanzan a la legitimidad del período de prueba ${ }^{21}$.

\section{Despido por absentismo}

Un recorrido parecido ha seguido la valoración del despido por absentismo a la luz de las normas internacionales. Aunque esta causa de despido objetivo arranca del RDLRT de 1977, en su versión estatutaria original el art. 52.d) ET le fijó una serie de requisitos numéricos que la hicieron caer en cierto olvido. Sin embargo, tras su aligeramiento por las reformas de 2010 y 2012, adquirió un protagonismo que no había tenido con anterioridad. Sin entrar en mucho detalle ${ }^{22}$, el cuestionamiento del precepto se ha basado en que permite establecer una cierta conexión causal entre la enfermedad del trabajador y su despido. Aunque el Tribunal Supremo no había encontrado nada extraño en ella y venía aplicándolo con normalidad ${ }^{23}$, se plantearon cuestiones prejudiciales en el plano constitucional y en el ámbito comunitario. Las mismas fueron contestadas, por orden cronológico, por las STJUE 18 enero 2018, C-270/16, Ruiz Conejero, y STC 118/2019, de 16 de octubre, que aceptaron ambas la legitimidad del precepto cuestionado, aunque la primera lo hiciera con algunos matices.

\footnotetext{
${ }^{19}$ Para un análisis de los primeros pronunciamientos en este terreno, véase SALCEDO BELTRÁN, M.C., "Jurisprudencia del Comité Europeo de Derechos Sociales y período de prueba del Contrato de Apoyo a Emprendedores: La aplicación del control de convencionalidad en España”, Lex Social. Revista jurídica de los derechos sociales, vol. 4, 2(2014), pp. 29 ss.

${ }^{20}$ En este sentido, entre otras, SSTSJ Andalucía (Málaga) 4 diciembre 2019, rec. 1268/2019, Cataluña 1 marzo 2016, rec. 6039/2015, 20 diciembre 2018, rec. 5235/2018, 13 diciembre 2019, rec. 5209/2019, Com. Valenciana 23 octubre 2018, rec. 2678/2018, o Madrid 11 noviembre 2019, rec. 958/2019, 22 mayo 2020, rec. 1393/2019.

${ }^{21}$ Incorporan literalmente el voto particular las SSTSJ Canarias (Las Palmas) 28 enero 2016, rec. 581/2015, 30 marzo 2016, rec. 989/2016, 18 abril 2016, rec. 110/2016, 25 noviembre 2016, rec. 894/2016, 31 enero 2017, rec. 1300/2016, 7 junio 2019 , rec. 367/2019, 31 julio 2019, rec. 488/2019, 16 julio 2020, rec. 383/2020, o Castilla-León (Valladolid) 19 diciembre 2016, rec. 2099/2016. En la misma línea SSTSJ Galicia 26 abril 2019, rec. 4258/2018, y 8 mayo 2020, rec. 168/2020.

${ }^{22}$ Para un análisis detallado de los diferentes episodios judiciales, véase, recientemente, MORENO GENÉ, J., "La extinción del contrato por absentismo: productividad empresarial versus derechos constitucionales reconocidos al trabajador", Revista General de Derecho del Trabajo y de la Seguridad Social 55(2020).

${ }^{23}$ En esta línea, por ejemplo, la STS 700/2017, de 21 septiembre, había descartado que el absentismo pudiera equipararse a discapacidad a efectos de aplicar la directiva.
} 
Inicialmente, la intervención de TJUE y TC pareció cerrar la cuestión. Varios TTSSJJ, incluyendo el de Cataluńa, incorporaron la doctrina establecida por la STC 118/2019 ${ }^{24}$. Sin embargo, la STSJ Cataluña 17 enero 2020, rec. 5532/2019 -seguida inmediatamente la de 28 febrero 2020, rec. 5885/2019- introdujo un sustancial vuelco en el panorama interpretativo. Se trata de una sentencia conocida y comentada en la que no entraré con mucho detalle ${ }^{25}$. El TSJ valora la posibilidad de despedir por "faltas de asistencia al trabajo, aun justificadas pero intermitentes" que abría el art. 52.d) ET a la luz de una amplísima gama de normas internacionales. A la postre, tras una extensísima argumentación, el precepto legal se declara inaplicable sobre la base de los arts. 6 convenio 158 OIT -cuyas salvedades se orillan por medio de su reinterpretación a la luz de del no ratificado art. 24 CSE revisada-, 4 convenio 155 OIT y 11 de la Convención sobre la Eliminación de toda forma de Discriminación contra la Mujer.

Dado que el precepto objeto del control de convencionalidad fue derogado inmediatamente después (RDL 4/2020, de 18 de febrero), no vale la pena detenerse en una discusión minuciosa de su argumentación. Sí considero importante, sin embargo, señalar que los diferentes argumentos que se emplean, relacionados con la protección frente al despido, los riesgos para integridad física y salud derivados de la posible valoración de ausencias de enfermedad y la posible discriminación indirecta por razón de género habían sido objeto de discusión en el Tribunal Constitucional, aunque en relación con los correspondientes preceptos de nuestra Constitución. La mayoría optó por descartarlos si bien se incorporaron a los diferentes votos particulares de la STC 118/2019, de 16 de octubre. Por otro lado, una parte de las cuestiones vinculadas al control de convencionalidad, fueron igualmente objeto de atención en el pronunciamiento constitucional al hilo de la consideración del derecho al trabajo: aunque recordó su doctrina de que "los tratados internacionales no integran el canon de constitucionalidad bajo el que hayan de examinarse las leyes internas..." de modo que "la eventual contradicción entre la regulación interna y los convenios y tratados internacionales ratificados por España... (es) un juicio de aplicabilidad -control de convencionalidad- que pertenece al ámbito de la legalidad ordinaria", añadió adicionalmente que no apreciaba "que la regulación contenida en el art. 52 d) LET contradiga lo dispuesto en el párrafo 1 del art. 6 del Convenio 158 de la OIT" habida cuenta las previsiones del párrafo 2 del precepto fundamentarían suficientemente la actuación del legislador interno. La sala sentenciadora del TSJ Cataluńa consideró, sin embargo, que estas consideraciones eran meros obiter dicta que no le vinculaban, al emitirse fuera de la competencia propia del TC, limitada al campo de la constitucionalidad.

\section{¿Un cuestionamiento integral de la regulación interna de los efectos del despido?}

Aun podría traer algún ejemplo adicional. Recientemente hemos conocido la STSJ Baleares 18 noviembre 2020 (rec. 179/2020) que, con base en el convenio 158 OIT, admite la impugnación individual del despido colectivo desde la perspectiva causal en caso de existencia de acuerdo en las consultas, aun a pesar de que el TS en su sentencia 699/2018, de 2 julio, la había descartado. Pero creo que, para concluir estas reflexiones sobre episodios concretos, es mejor alzar un poco la mira y observar a vista de pájaro la posible existencia de un objetivo más amplio: la propia regulación de

\footnotetext{
${ }^{24}$ Cfr. SSTTSSJJ Castilla-La Mancha 17 diciembre 2019, rec. 823/2019, Castilla-León (Burgos) 20 diciembre 2019, rec. 745/2019, y 29 enero 2020, rec. 14/2020, Cataluña 10 enero 2020, rec. 5547/2020, o Extremadura 23 enero 2020, rec. 656/2019. En cuanto al TSJ Cataluña, cabe notar que el precepto en cuestión se ha aplicado con posterioridad a la inflexión interpretativa que se describe a continuación en el texto (cfr. sentencia 24 julio 2020, rec. 1313/2020).

${ }^{25}$ Para un análisis de su contenido, véanse, por ejemplo, FORCADELL, NAVARRO, "Compatibilidad" cit. o CIALTI, P.-H., "El despido del art. 52 d) no supera(ba) el control de convencionalidad", Temas laborales 152(2020).
} 
los efectos del despido. Desde el RDLRT de 1977, se introdujo un régimen de indemnizaciones tasadas como reacción típica frente al despido injusto y solo excepcionalmente se prevé la obligación de readmisión forzosa; y este modelo continúa presente aun hoy en las reglas estatutarias, con independencia de los cambios que han sufrido a lo largo de los años los criterios de cuantificación de las compensaciones o los supuestos en los que procede la ejecución in natura. Sin embargo, en los últimos tiempos, parece estar siendo puesto en cuestión a la luz de las normas internacionales.

Probablemente la cuestión más interesante, por referirse a un elemento clave del equilibrio del ordenamiento laboral desde hace casi medio siglo, se relaciona con el tratamiento del despido sin causa. Dado que el sistema implica que la voluntad unilateral del empresario supone por sí misma la extinción del vínculo con la única condición de que se abone la indemnización establecida, puede pensarse que ello equivale al reconocimiento del tan temido despido libre. Aunque por supuesto ello no es así, la apreciación está probablemente en la base de los cuestionamientos judiciales que afectan al modelo de cuando en cuando. Si a mediados de la década de los 80 se intentó cambiar mediante la creación judicial de los despidos nulos por fraude de ley, el resultado se persigue ahora a través de la discusión de las normas legales que lo configuran a la luz de los imperativos que se deducirían de las normas internacionales. Habida cuenta que la proyección del fraude de ley con la finalidad de obtener la nulidad del despido sin causa parece firmemente cerrada en la jurisprudencia ordinaria ${ }^{26}$, hemos asistido en los últimos meses al intento de reabrir la cuestión al amparo de las normas internacionales aplicables. Hasta el momento, los Tribunales Superiores no han sido proclives a aceptar el planteamiento ${ }^{27}$, aunque cabe señalar que, en la sala de Cataluña, reunida de forma plenaria, si bien la mayoría se ha situado en la misma línea, se ha formulado un extenso voto particular firmado por varios magistrados ${ }^{28}$. La cuestión, pues, no parece enteramente cerrada.

Como tampoco lo está, por otro lado, la cuestión de la legitimidad de otros aspectos de la regulación española de los efectos del despido. Así, por lo que se refiere al sistema de indemnizaciones tasadas establecido en nuestro ordenamiento, aunque el Tribunal Constitucional descartó que el mismo resultara contrario a los preceptos de nuestra constitución ${ }^{29}$, ha sido reabierto en algún pronunciamiento de instancia reciente con base en las normas internacionales ${ }^{30}$. Como ha sido cuestionado en instancia la adecuación a las mismas de la titularidad empresarial de la opción entre indemnización y readmisión ${ }^{31}$. De hecho, a la vista de algún comentario a una de las sentencias que hemos considerado en este apartado, las normas internacionales suministrarían herramientas para un completo programa de revisión de nuestra legislación sobre despido ${ }^{32}$. Y, a juzgar por los pronósticos doctrinales, cabe prever que la anunciada ratificación de la Carta Social Europea revisada podría desplegar efectos adicionales en este terreno ${ }^{33}$.

\footnotetext{
${ }^{26}$ STS 944/2017, de 29 noviembre 2017, con cita de otras anteriores.

${ }^{27}$ Véanse SSTSJ Andalucía (Sevilla) 19 noviembre 2020, rec. 1795/2019, Castilla-León (Valladolid) 16 noviembre 2020, rec. 1699/2020, y sobre todo, Madrid 3 noviembre 2020, rec. 587/2020, que afronta extensamente el problema.

${ }^{28}$ STSJ Cataluña 21 julio 2020, rec. 4708/2020.

${ }^{29}$ Cfr. AATC 43/2014, 12 febrero, y 35/2015, 17 febrero.

${ }^{30}$ SJS Barcelona-26 170/2020, 31 julio.

${ }^{31}$ SJS Madrid-34 71/2020, 21 de febrero.

${ }^{32}$ FORCADELL, y NAVARRO, "Compatibilidad del control de convencionalidad" cit., pp. 152 ss.

${ }^{33}$ Sobre el impacto que puede tener en algunas de las cuestiones que han sido objeto de análisis, véanse MOLINA NAVARRETE, C., "Adiós al despido por absentismo justificado: expulsando, látigo jurídico en mano, a los mercaderes del templo de la salud, pero sin regla de derecho intertemporal y sin prohibición causal" (https://www.laboral-social.com/adios-despido-absentismo-justificadordl-4-2020.html; consulta 18 enero 2021), BAYLOS, A.P., “¿Debe ser declarado improcedente el despido por enfermedad tras la derogación del art. 52.d) ET?” (https://baylos.blogspot.com/2020/02/debe-ser-declarado-improcedente-el.html; consulta 18 enero 2021) y, más recientemente, BELTRÁN DE HEREDIA RUIZ, I., "STSJ AndISevilla 19/11/20: el despido con causa falsa es improcedente (y una reflexión sobre el art. 24 Carta Social Europea)” (https://ignasibeltran.com/2020/11/23/stsj-andsevilla-19-11-20-eldespido-con-causa-falsa-es-improcedente-y-una-reflexion-sobre-el-art-24-carta-social-europea/; consulta 18 enero 2021).
} 


\section{¿Activismo o autocontención?}

Los episodios comentados muestran como la (histórica) admisión y el (reciente) descubrimiento de lo que se ha dado en llamar control de convencionalidad pone un extraordinario poder en manos de los órganos de la jurisdicción ordinaria. Ya no son solo "la boca que pronuncia las palabras de la ley, seres inanimados que no pueden mitigar la fuerza y el rigor de la ley misma," como postulara Montesquieu, sino que el control de convencionalidad les abre la posibilidad de desplazarla. Es claro, por supuesto, que este poder se asienta en bases diferentes a las que sustentan el poder legislativo y, por tanto, tiene condicionantes distintos. Ahora bien, aunque se trata de una obviedad, me parece que ello no siempre se percibe en la valoración del modo en que una parte de los jueces están aplicando la convencionalidad. Los epítetos que se utilizan para hacerla presuponen, según creo, la participación de la voluntad en su conformación ${ }^{34}$. Y esto me parece preocupante, no porque sea un ingenuo que considere que los procesos interpretativos se basan en exclusiva en la lógica jurídica, sino porque el imperio de la ley (arts. 117.1 CE y 1 LOPJ) sigue siendo un elemento central en la configuración del Estado de Derecho.

Entiendo en este sentido, que, aun aceptando que las operaciones de interpretación no son abstractas ni ideológicamente neutras e incorporan, por tanto, dosis de subjetividad y voluntad de sus protagonistas, la prevalencia de la ley, como concreción del principio de mayoría, hace que no pueda admitirse cualquier posible interpretación. La exigencia de cierto rigor en la aplicación de categorías y principios interpretativos resulta imprescindible si se quiere evitar que el control de convencionalidad se transforme en "un irreflexivo activismo judicial que aboque a una suerte de "gobierno de los jueces» o una "juristocracia»"35. Una situación así tiene, en primer lugar, un elevado coste en términos de inseguridad jurídica, que, además, no juega en forma unidireccional. Esto último lo acabamos de ver en relación con la prohibición de despedir durante la pandemia (art. 2 RDL 9/2020): el control de convencionalidad puede operar no solo para recuperar los estándares de protección que se habrían perdido con ocasión de las pasadas reformas laborales sino para vaciar de contenido los nuevos ${ }^{36}$. Pero, en segundo lugar, y sobre todo, implica una profunda alteración de las diferentes funciones y, sobre todo, de los distintos fundamentos y legitimidades de los poderes del Estado. Después de todo, aunque "la justicia emana del pueblo" (art. $117 \mathrm{CE}$ ), la legitimación democrática de los órganos judiciales es distinta e inferior a la de las Cortes Generales que "representan al pueblo español" (art. $66 \mathrm{CE}$ ).

En este contexto, creo que las exigencias de profundizar en el sistema multinivel de protección de los derechos sociales, de incorporar con normalidad el control de convencionalidad y de preservar la prevalencia de las normas internacionales en los términos del art. 96.1 CE, deben ser cohonestadas con el imperio de la ley como criterio esencial de funcionamiento del ordenamiento interno. Y para ello se precisa la adopción de una actitud de autocontención por parte de los órganos judiciales llamados a hacer efectivo el control difuso de convencionalidad. Autocontención (self-restraint) u otros términos similares son nociones que se han utilizado para hallar un punto razonable de equilibrio entre las formidables atribuciones de los jueces para garantizar la adecua-

\footnotetext{
${ }^{34}$ En esta línea, alguna de las sentencias que se ha considerado en el apartado anterior ha sido calificada como "valiosa y también valiente” (BAYLOS, A.P., “¿Debe ser declarado improcedente?” cit.) o "revolucionaria” (FORCADELL, y NAVARRO, “Compatibilidad del control de convencionalidad” cit., p. 155), adjetivos que presuponen más la voluntad que la inferencia lógica. Se ha afirmado asimismo que la aplicación de las normas internacionales del trabajo "no deja de ser una cuestión o una práctica que queda en buena medida en manos de la propia voluntad y actitud del juzgador, contribuyendo a esa indeseada inseguridad jurídica de la que tanto se habla" (MARTÍNEZ MORENO, "El marco internacional” cit., p. 114).

${ }^{35}$ La cita es de JIMENA, "Jurisdicción nacional y control de convencionalidad" cit., p. 164.

${ }^{36}$ Me refiero a la SJS Barcelona-1 283/2020, 15 diciembre, que opta por inaplicar la prohibición de despidos por causas sobre la base del reconocimiento de la libertad de empresa por la CDFUE.
} 
ción de las leyes a la constitución, sean difusas o concentradas según las respectivas tradiciones, y la evitación de la asunción de facultades de creación normativa que corresponden al poder legislativo; y bien puede ser utilizado para encontrarlo en el problema que nos ocupa ${ }^{37}$. Por supuesto, la afirmación de esta idea no resulta suficiente para cerrar el tema definitivamente. Ello requeriría un análisis minucioso de esta noción así como una revisión exhaustiva de las implicaciones que su proyección tendría sobre las diferentes normas internaciones. Y estas tareas desbordan, con toda probabilidad, mis capacidades e, indudablemente, el espacio del que dispongo. Lo que sí puedo, sin embargo, es individualizar algunos aspectos que deberían ser considerados para alcanzar el objetivo de una adecuada aplicación de las normas internacionales que, sobre la base de la autocontención, respetara imperio de la ley y principio de seguridad jurídica. Algunos son de carácter procedimental y otros, sustantivos.

Por lo que se refiere a los primeros, mirarían, de un lado, a la garantía de los derechos de defensa y, de otro, a la minimización de las contradicciones dentro del sistema. Frente a ellos, la lectura de las sentencias que he considerado con anterioridad permite detectar determinadas manifestaciones de voluntarismo procedimental que ponen en riesgo aquellos e incrementan la entropía dentro del sistema jurídico. A veces, el control de convencionalidad parece suscitarse con cierto quebranto de los principios procesales. Vale como ejemplo la conocida y ya citada STSJ Cataluña de 17 enero 2020, rec. 5532/2019, en relación con la inaplicación del art. 52.d) ET por vulneración de diferentes normas internacionales, cuya lectura parece mostrar un desconocimiento por la sala sentenciadora del carácter extraordinario del recurso de suplicación y sus consecuencias ${ }^{38}$. En todo caso, el principal aspecto a comentar se relaciona con los criterios para la selección de la norma aplicable para verificar la legal a la luz de los imperativos de los derechos sociales que no siempre apunta en la línea de la armonía sistemática. Dado que el sistema de protección de los derechos humanos es multinivel, es lógico que para cada aspecto en controversia pueda existir una norma

\footnotetext{
${ }^{37}$ En relación con este concepto, GARCÍA DE ENTERRÍA, E., "La posición jurídica del Tribunal Constitucional en el sistema español: posibilidades y perspectivas" (1981), reimpresión en Revista Española de Derecho Constitucional 100(2014), pp. 124 ss. Ha sido utilizado también en el ámbito de la jurisprudencia del TEDH como instrumento para garantizar las peculiaridades existentes a nivel nacional: cfr. MARTÍNEZ ESTAY, J.I., "Auto-restricción, deferencia y margen de apreciación. Breve análisis de sus orígenes y su desarrollo", Estudios Constitucionales, 1 (2014), pp. 365 ss.; para una crítica, GARCÍA ROCA, J., "La muy discrecional doctrina del margen de apreciación nacional según el TEDH: soberanía e integración”, Teoría y realidad Constitucional 20(2007), pp. 117 ss.

${ }^{38}$ En este sentido, llamo la atención sobre el limitado alcance del recurso planteado por la parte demandante y recurrente. Literalmente, la sentencia indica al respecto:
}

"Al amparo del art.191c) de la Ley 31/11 (sic), en realidad art.193c) Ley 36/11 (LRJS), la recurrente denuncia la infracción del art.54d ET (en realidad art.52.d) ET), al considerar que las ausencias justificadas en que se basa el despido de la trabajadora no reúnen el carácter de intermitentes, con cita de la STS 7/05/2015. En apoyo de tal tesis, la recurrente sostiene que la ausencia del 26/11/18 al 5/12/18 que supuso la reincorporación el 10/12/18 con una duración de 15 días naturales no tiene el carácter de intermitente".

Frente a este único motivo de suplicación, la Sala opta por dar una larga cambiada y suscita como "la aplicabilidad de la norma al caso" como "cuestión previa", lo que le permite entrar a valorar el art. 52.d ET a la luz de las normas internacionales. Es verdad que este planteamiento podría ser aceptado en aplicación del principio iura novit curia. Pero no es muy frecuente en el ámbito del recurso de suplicación, que no puede construirse de oficio por el órgano ad quem y cuyo carácter extraordinario impide suscitar cuestiones nuevas. No es de extrañar en esta línea que la posterior STSJ Cataluña 17 septiembre 2020, rec. 918/2020, opte por dar una solución diferente a un caso sustancialmente igual por imperativo de la congruencia:

"habiéndose pronunciado la STC 118/2019 de 16 de octubre, declarando la constitucionalidad del artículo 52.d.) del ET, y no habiéndose planteado en momento alguno por las partes en este procedimiento el eventual análisis de convencionalidad de la norma, analizado por esta Sala en Sentencias n o 274/2020, de 17 de enero y 1142/2020, de 28 de febrero, ciñéndonos a los términos en que aparece formulada la demanda, el recurso de suplicación y su impugnación, limitados a determinar la interpretación del requisito "días consecutivos" del artículo 52.d.) del ET, por las razones anteriormente expuestas y en aplicación de la doctrina unificada del TS mencionada, debe ser estimado el recurso y declarado procedente el despido impugnado".

Es singularmente llamativo que dos componentes de la sección que dicta esta segunda sentencia participaron en la que deliberó la primera. 
diferente en cada uno de los planos en los que se descompone. Ahora bien, algunos de estos planos tienen su propio mecanismo de garantía cuya decisión es vinculante para los órganos judiciales. En este contexto, el voluntarismo puede manifestarse mediante la selección por el órgano judicial del fundamento para la eventual rectificación de la norma legal aplicable, lo que permite incrementar las propias facultades en vistas a su desplazamiento ${ }^{39}$. A mi juicio, esta decisión no debería ser libre para el juez. Después de todo, conforme al art. 10.2 CE, "las normas relativas a los derechos fundamentales y a las libertades que la Constitución reconoce se interpretarán de conformidad con la Declaración Universal de Derechos Humanos y los tratados y acuerdos internacionales sobre las mismas materias ratificados por España", lo que apuntaría hacia un papel prevalente de la jurisdicción constitucional también para protagonizar el diálogo judicial en el conjunto del sistema de protección de los derechos fundamentales. Es verdad que, aunque esta solución es adecuada a la evolución del sistema europeo de protección de derechos humanos y tiene importantes defensores teóricos, no es segura en términos formales ${ }^{40}$. Sin embargo, parece razonable pensar que en aras a la preservación de la seguridad jurídica los diferentes componentes del sistema deben ser utilizados en forma ordenada. Aunque sea solo por sentido común, en aras a la preservación de la supremacía de la ley, diría que el control judicial directo basado en la convencionalidad aparece como una vía subsidiaria a otros elementos que puedan servir a la misma finalidad.

Desde un punto de vista sustantivo, creo que la autocontención impone un análisis textual cuidadoso de los documentos generados en el derecho internacional, tanto por lo que se refiere a su contenido como en relación con su naturaleza. Desde la primera perspectiva, es tradicional la exigencia de que las normas contenidas en acuerdos internacionales han de ser self-executing para poder ser objeto de aplicación directa. Es verdad que, conforme al art. 30.1 Ley 25/2014, parece existir una suerte de presunción favorable a que tienen tal carácter puesto que, como regla general, "los tratados internacionales serán de aplicación directa", y este criterio solo cederá cuando "de su texto se desprenda que dicha aplicación queda condicionada a la aprobación de las leyes o disposiciones reglamentarias pertinentes". Con esto y con todo, el juego de este criterio favorable a la aplicación directa obliga a un examen cuidadoso de las reglas establecidas pues no parece fácil aceptar la eficacia directa de normas que se formulan como amplios principios ni de aquellas que se insertan en un contexto del que se desprende la existencia de diferentes posibilidades de concreción por los Estados firmantes.

La casuística judicial reciente no siempre se acomoda a este patrón: aparte los casos en los que se han traído a colación normas internacionales que no han sido objeto de ratificación ${ }^{41}$, obser-

\footnotetext{
${ }^{39}$ Esta es una de las razones por las que CRUZ VILLALÓN, J., "El despido injustificado por COVID” (http://jesuscruzvillalon. blogspot.com/2021/01/el-despido-injustificado-por-covid-la.html; consulta 12 febrero 2021), considera "profundamente" errónea la citada SJS Barcelona-1 283/2020, 15 diciembre, en relación con el art. 2 RDL 9/2020, al haberse basado en el art. 16 CDFUE y descartado plantear cuestión de inconstitucionalidad con base en el art. 38 CE si consideraba que la regla vulneraba la libertad de empresa. Pero, esto mismo se encuentra en algunos otros de los supuestos que hemos considerado en el trabajo. Por ejemplo, en la citada STSJ Baleares 18 noviembre 2020 (rec. 179/2020), en relación con la impugnación individual del despido colectivo: aunque en el motivo de suplicación se denunció "vulneración del derecho fundamental a la tutela judicial efectiva consagrado en el artículo 24 de la Constitución Española (CE) y en el artículo 47 Carta de Derechos Fundamentales de la Unión Europea”, el asunto se hace desembocar en el control de convencionalidad con base en el convenio 158 OIT. Ello permite rectificar la doctrina previa del TS, sin necesidad de plantear cuestión ante TC o ante TJUE. Lo mismo cabría decir, en fin, de los pronunciamientos del TS sobre el alcance del control causal. Al fundamentarse en el convenio 158 OIT se orilla la necesidad de plantear cuestión de constitucionalidad ante el TC, a pesar de que el derecho al trabajo se consideraba también afectado por la decisión legislativa.

${ }^{40} \mathrm{Al}$ respecto, véanse CANOSA, "El control" cit., o LÓPEZ GUERRA, L., "Convencionalidad y constitucionalidad: los casos de México y España”, Boletin Mexicano de Derecho Comparado, 2019, pp. 43 ss. Las dudas sobre la viabilidad de la reconstrucción así como las propuestas para su rearfimación, en CANOSA, pp. 124 ss.

${ }^{41}$ Es el caso de la Carta Social Europea revisada en la STSJ Cataluña de 17 enero 2020, rec. 5532/2019: a pesar de la falta de ratificación se convierte en "innegable criterio hermenéutico" que permite evitar los problemas que derivan del art. 6 convenio 158 OIT a la hora de justificar la inadecuación a este del art. 52.d) ET pues permite reconvertir su alcance al "garantizar un estándar de protección más elevado".
} 
vamos como en ocasiones parece asociar eficacia directa a normas que contienen meros principios o que incluyen condicionantes y reservas que no son susceptibles de integración judicial. En el primer sentido, vale como ejemplo la asociación de diferentes normas internacionales al control de convencionalidad en relación con la temática de la conciliación de la vida laboral y familiar ${ }^{42}$; en el segundo, la eficacia incondicional que se asigna a los convenios de la OIT, señaladamente, aunque no solo, al núm. 158 en materia de terminación del contrato, sin tener en cuenta las múltiples salvedades y reservas que contienen sus reglas ${ }^{43}$ y que oportunamente fueron valoradas en momentos anteriores por la jurisprudencia. Es interesante, en este último sentido, releer las SSTS dictadas en relación con los primeros conflictos en los que se planteó la aplicación del convenio 158, en concreto su art. 7. Si bien es cierto que el TS partió inicialmente de un apriorismo sobre el carácter no self-executing del conjunto del convenio ${ }^{44}$, en un momento posterior la decisión se basó más bien en las dificultades para reconocer que tuviera tal carácter la concreta regla internacional cuya aplicación se postulaba pues "las técnicas de flexibilidad empleadas en su formulación permiten... diversas posibilidades de desarrollo y un amplio sistema de excepciones, no sólo subjetivas por la vía del artículo 2 , sino, sobre todo, objetivas, en atención al criterio de razonabilidad de la negativa empresarial a la audiencia" 45 .

Desde el segundo punto de vista, creo que hay que ser igualmente cauto en relación con lo que podríamos llamar «documentación interpretativa» emitida en el ámbito internacional. Como se ha indicado, aparte las normas convencionales en sentido estricto, el sistema social multinivel incluye mecanismos de naturaleza variada que emiten criterios interpretativos en relación con aque-

${ }^{42}$ El listado de tratados utilizado por la saga de sentencias abierta por STSJ Canarias (Las Palmas) 12 marzo 2019, rec. 19/2019, es muy amplio. Su asociación al control de convencionalidad, sin embargo, es discutible. Se citan reglas de la Convención sobre la eliminación de todas las formas de discriminación de las mujeres (art. 11), del convenio OIT núm. 156 sobre los trabajadores con responsabilidades familiares y de la CSE (art. 8.3). La lectura de estos preceptos permite observar muchas expresiones que implican su carácter de principios que iluminan la tarea legislativa en los Estados firmantes.

${ }^{43}$ Valga, como ejemplo, de un lado, la utilización del convenio 158 de la OIT para justificar la corrección de la literalidad legal en materia de control causal. Es verdad el convenio establece un principio de causalidad en la terminación de la relación de trabajo por decisión empresarial (art. 4) que obliga a que existan organismos nacionales que puedan "examinar las causas invocadas para justificar la terminación de la relación de trabajo y todas las demás circunstancias relacionadas con el caso, y para pronunciarse sobre si la terminación estaba justificada" (art. 9.1). Pero no lo es menos que el posterior art. 9.3 contiene una regla especial para el control de los despidos basados en "necesidades de funcionamiento de la empresa, establecimiento o servicio". De acuerdo con ella, en este tipo de despidos los indicados organismos "estarán facultados para verificar si la terminación se debió realmente a tales razones, pero la medida en que esos organismos estarán facultados también para decidir si esas razones son suficientes para justificar la terminación deberá determinarse por los métodos de aplicación mencionados en el artículo 1 de este Convenio". Como ha advertido una parte de la doctrina (GIL PLANA, J., "Despidos colectivos: aspectos sustantivos", en MONTOYA, A., y GARCÍA MURCIA, J. (dirs.), Comentario a la reforma laboral de 2012, Madrid (Civitas), 2013, pp. 253 ss. p. 266; JURADO SEGOVIA, A., "Reforma en materia de las causas de despido colectivo y objetivo", en THIBAULT, J. (dir.), La reforma laboral de 2012: nuevas perspectivas para el derecho del trabajo, Madrid (La Ley), 2012, pp. 479 ss,), este último precepto se mueve en la línea de posibilitar que la legislación y práctica nacionales determinen en cada estado firmante el alcance que corresponde al control: siendo inexorable el de existencia, el de "suficiencia” puede establecerse o no, y en el caso afirmativo, en mayor o menor medida. En esta línea, la documentación elaborada en el seno de la propia OIT pone de manifiesto que este precepto "permite, en consecuencia, que cada país limite los poderes que tiene el organismo competente, cuando examina si la terminación está justificada, de ejercer un control sobre la decisión del empleador en cuanto al número de trabajadores de su empresa" (OIT, Protección contra el despido injustificado, 1995, apartado 214).

De otro lado, en relación con la presunta incompatibilidad entre el derogado art. 52.d) ET y el art. 6 de este convenio, el TC había afrontado el tema de la posible contradicción del art. 52.d) ET con las normas internacionales suscritas por España. En este terreno, recordó su doctrina de que "los tratados internacionales no integran el canon de constitucionalidad bajo el que hayan de examinarse las leyes internas..." de modo que "la eventual contradicción entre la regulación interna y los convenios y tratados internacionales ratificados por Espańa... (es) un juicio de aplicabilidad — control de convencionalidad — que pertenece al ámbito de la legalidad ordinaria", para añadir adicionalmente que no apreciaba "que la regulación contenida en el art. $52 \mathrm{~d}$ ) LET contradiga lo dispuesto en el párrafo $1 \mathrm{del}$ art. 6 del Convenio 158 de la OIT" habida cuenta las previsiones del párrafo 2 del precepto fundamentarían suficientemente la actuación del legislador interno (véase también en esta línea STSJ Madrid 2 diciembre 2013, rec. 1190/2013).

${ }^{44}$ SSTS 4 y 5 noviembre 1987. RJ 1987/7810 y RJ $1987 / 781$.

${ }^{45}$ STS 8 marzo 1988. RJ 1988\1885. 
llas. Es posible que algunos de ellos puedan llegar a tener efectos directos en el ámbito interno, pero seguramente existen otros que no. Nuestro ordenamiento lo deja claro para los pronunciamientos del TJUE -aunque no sería estrictamente necesario habida cuenta el desarrollo del derecho de la Unión Europea- y del TEDH -a pesar de que este efecto no resulta directa ni evidentemente del $\mathrm{CEDH}-^{46}$. Para los restantes órganos existentes en el ámbito internacional que generan documentos con vocación interpretativa o integrativa de los tratados será preciso un minucioso análisis que permita elucidar si y en qué condiciones pueden ser proyectados directamente en el ámbito interno mediante su incorporación al control de convencionalidad.

Entiendo, a la postre, que es preciso ser consciente de que las cautelas a la hora de aplicarlo tienen precisas valencias para el ordenado funcionamiento del sistema jurídico. Se ha indicado en este sentido que la adaptación judicial del ordenamiento interno a las exigencias internacionales, aparte problemas de inseguridad, no permite una valoración de conjunto de los intereses en juego $^{47}$. Como seńalara el TS en la década de los 80 , en relación con los primeros asuntos en los que se buscaba la aplicación directa del convenio 158 OIT, "sólo la ley puede abordar con la necesaria generalidad y precisión” la adaptación del ordenamiento interno a las reglas internacionales ${ }^{48}$. Ello no impide, por supuesto, que en casos de evidente contradicción entre estas y aquella sea necesario recurrir a su desplazamiento. Pero, antes de tomar tan trascendente decisión, el juez debería intentar recordar cuándo fue la última vez que los ciudadanos acudimos a votarlo.

\footnotetext{
${ }^{46}$ Cfr., con carácter general, arts. 4 bis y 5 bis LOPJ; en el ámbito social, art. 219.2 LRJS

${ }^{47}$ En este sentido, a propósito del impacto de la posible ratificación de la CSE revisada, CRUZ, "El despido injustificado" cit. Muy interesante la reflexión en el plano metafórico de BELTRÁN DE HEREDIA, I., "Indemnización por despido improcedente, Darwin y el sesgo de supervivencia” (https://ignasibeltran.com/2021/02/09/indemnizacion-por-despido-improcedente-darwin-y-elsesgo-de-supervivencia/: consulta 12 febrero 2021).

${ }^{48}$ STS 8 marzo 1988. RJ 1988\1885.
} 